\title{
Correction to: Preparation of Controlled-Release Particles Based on Spherical Porous Silica Used as the Drug Carrier by the Dry Coating Method
}

\author{
Shohei Nakamura, ${ }^{1,2}$ Shihoko Kondo, ${ }^{1}$ Ayaka Mohri, ${ }^{1}$ Takatoshi Sakamoto, ${ }^{1}$ and Hiroshi Yuasa ${ }^{1}$
}

\section{Correction to: AAPS PharmSciTech}

https://doi.org/10.1208/s12249-018-0958-7

In the present notation, the formula names and the formulas (page 7, left column, lines 20-21) do not correspond to each other. It is a completely incorrect description, due to a typesetting mistake by the publisher. See below for details. The original article has been corrected.

Incorrect notation

$$
\begin{array}{ll}
\mathrm{M}_{t} / \mathrm{M}_{\infty}=K t^{1 / 2} & \text { Zero-order model } \\
\mathrm{M}_{t} / \mathrm{M}_{\infty}=K t^{n} & \text { Korsmeyer-Peppas model }
\end{array}
$$

\section{Correct notation}

$\begin{array}{ll}\mathrm{M}_{t} / \mathrm{M}_{\infty}=K t & \text { Zero-order model } \\ \mathrm{M}_{t} / \mathbf{M}_{\infty}=K t^{1 / 2} & \text { Higuchi model } \\ \mathbf{M}_{t} / \mathbf{M}_{\infty}=K t^{n} & \text { Korsmeyer-Peppas model }\end{array}$

The online version of the original article can be found at https:// doi.org/10.1208/s12249-018-0958-7

\footnotetext{
${ }^{1}$ Department of Pharmaceutical Technology, College of Pharmaceutical Sciences, Matsuyama University, 4-2 Bunkyo-cho, Matsuyama, Ehime 790-8578, Japan.

${ }^{2}$ To whom correspondence should be addressed. (e-mail: snakamur@g.matsuyama-u.ac.jp)
} 